\title{
Thermal Infrared Enthalpimetry in Paper Microzone Plates for Green and High Throughput Determination of Wine Acidities
}

\author{
Daniele F. Ferreira, ${ }^{a}$ Bruna Tischer, ${ }^{b}$ Alexandre J. Cichoski, ${ }^{a}$ Cristiano R. Menezes, ${ }^{a}$ \\ Roger Wagner, ${ }^{a}$ Adilson B. Costa ${ }^{c}$ and Juliano S. Barin ${ }^{\circledR} * a$ \\ ${ }^{a}$ Departamento de Tecnologia e Ciência dos Alimentos, Universidade Federal de Santa Maria, \\ 97105-900 Santa Maria-RS, Brazil \\ ${ }^{b}$ Instituto de Ciência e Tecnologia dos Alimentos, Universidade Federal do Rio Grande do Sul, \\ 91501-970 Porto Alegre-RS, Brazil
}
${ }^{c}$ Grupo de Pesquisa em Quimiometria, Programa de Pós-Graduação em Sistemas e Processos Industriais, Universidade de Santa Cruz do Sul, 96815-900 Santa Cruz do Sul-RS, Brazil

\begin{abstract}
The use of paper devices in chemical analysis has been increased due to their low cost, high surface area, portability, and biodegradability. A novel use of paper devices is proposed using thermal infrared enthalpimetry (TIE) for a rapid, green, and high throughput determination of wine acidities. The determination of total, fixed, and volatile acidities in red, white, and sparkling wine was carried out, and the results were compared with AOAC titration method. A multichannel pipette was used for reagent addition and an infrared camera for the temperature monitoring. The pipetting and temperature measurement conditions presented direct influence in the deviations among the measurements. The results obtained by TIE presented an agreement between 96 and $104 \%$ with the reference method (AOAC 962.12), for all samples. A high sample throughput was achieved reaching 480 samples $^{-1}$ for total acidity. A significant reduction in sample volume and reagent consumption was also obtained, with energy consumption up to 60 times lower in relation to the AOAC method. The association of TIE with a paper device provided a rapid, reliable and green method for the determination of acidities of wines.
\end{abstract}

Keywords: infrared thermal imaging, paper analytical devices, wine acidity, green chemistry, digital image

\section{Introduction}

Increasingly, there is a demand in chemical analysis that researchers follow green chemistry principles, which have led to the development of new environmentally-friendly processes. ${ }^{1}$ The major goal of green analytical chemistry is to minimize the use and disposal of harmful solvents, material, and energy consumption, provide inherent safety, step integration, and miniaturization, and increase the use of renewable and recyclable resources. ${ }^{2,3}$ In this context, an effective tool to meet such requirements is the use of paper-based analytical devices, ${ }^{4}$ which are considered a sustainable platform for chemical analysis that allows higher flexibility, portability, low cost, and reduction of the sample and solvent volume. ${ }^{5,6}$

*e-mail: juliano@ufsm.br
Paper microzone plates have been used as a greener (and lower cost) alternative to plastic microplates. The construction of these devices could be based on patterning the paper with isolated hydrophilic zones through the application of a hydrophobic barrier. One of the most promising methods for this purpose is wax printing, which allows fast, inexpensive, and easy production of devices. ${ }^{6-9}$ This process involves only two steps: the wax is printed on the surface of the paper, then melted by heat application. The wax diffuses within the paper, creating a complete hydrophobic barrier. Considering the high surface to volume ratio of paper microzones, they have been used to speed up sample preparation, as evaporation/concentration steps. ${ }^{4,10,11}$

Although these devices enable analyses in agreement to green analytical chemistry principles, in general the official methods used to perform quality control of food are not moving in the same direction. ${ }^{12}$ Acidity is considered 
a routine analysis in the food and beverage industry, as it is essential for monitoring the quality of products. In general, three types of acidity could be measured: total, fixed, and volatile. Volatile acidity could be related to the organic acids that are more easily vaporized than the nonvolatile (fixed) ones, and it is often obtained by calculating the difference between measured total and fixed acidities. These parameters have been determined in several food and beverages by titration, a time-consuming method that requires constant attention of analyst, and ultimately leads to a low throughput. ${ }^{12,13}$

In order to overcome these drawbacks, methods using image detection have been used as faster alternatives for acidity determination. The colorimetric determination of acidity using a paper-based platform has been proposed as a simple, low cost, and environmentally-friendly method. ${ }^{14,15}$ However, despite its feasibility for acidity analysis, some limitations can still be observed related to colorimetric indication in samples with high color intensity, as wines. Thermal infrared enthalpimetry (TIE) could be an effective alternative for the determination of acidities in cloudy or colored samples as it is based on infrared rather than visible detection. ${ }^{16-18}$ Using TIE, the analysis could be performed more quickly with a contactless sensor (e.g., infrared cameras) for monitoring of reactions commonly used in titrations (e.g., neutralization). In TIE, the direct injection enthalpimetry approach has been used, and a solution containing a reagent in stoichiometric excess is mixed with the sample solution. The heat released from the resulting reaction is proportional to the amount of analyte in the sample, and calibration curves have been constructed for calibration. ${ }^{16}$

In TIE, polymeric microplates have been used as reactors, and a multichannel pipette has been used for simultaneous addition of solutions to multiple wells. Several features have been reported, such as simplicity, rapidity, simultaneous detection of multiple reactions, and high throughput analysis. ${ }^{16}$ TIE was used to determine acidities in different vinegars and pickled vegetable brine with good agreement with conventional titration. ${ }^{17,18}$ Additionally, it can also be used to determine alcohol content from beverages and the saponification value of edible oils. ${ }^{19-21}$ Considering the applications in literature for TIE, a reduction in the reagent consumption/waste generation, as well as energy expenditure in one or two orders of magnitude, could generally be obtained in comparison with reference methods. However, TIE can become greener if paper microplates are used, reducing the amount of solvents, samples, and reagents required for analysis and the generation of residues for disposal. ${ }^{22}$
Considering the advantages of TIE and the features of paper microzones plates, they were combined for the development of a new method for the determination of acidities of wine (total, fixed, and volatile). The volume of solutions, dispensing rates of reagent in stoichiometric excess, and the number of concentration cycles required to perform the determination of fixed acidity were evaluated. The image processing was also evaluated considering the region of interest (ROI) required to obtain reasonable temperature values. Microscopies were used to show the surface of microzones with and without samples, and the results were compared with those obtained from the reference methods of analysis, ${ }^{12}$ and an evaluation of the parameters of merit of the proposed method was performed.

\section{Experimental}

\section{Samples and reagents}

Samples of red, white, and sparkling wine from three different manufactures were obtained from the local market. Calibration curves were constructed using reference solutions of tartaric acid (Vetec, Brazil), and sodium hydroxide (Dinâmica, Brazil) as reagent in stoichiometric excess. Standardization of the tartaric acid solutions was performed with sodium hydroxide that had previously been standardized with potassium biphthalate (Vetec, Brazil). Deionized water was further purified in a Milli-Q system (Direct-Q 3 UV, $18.2 \mathrm{M} \Omega \mathrm{cm}$, Millipore Corp., USA), and was then used to prepare all solutions. Qualitative filter paper (CA5011260, $60 \times 60 \mathrm{~cm}, 14 \mu \mathrm{m}$ of porosity, $80 \mathrm{~g} \mathrm{~m}^{-2}$ of grammage, Prolab, Brazil) and black wax ink (Xerox ColorQube, USA) were used to manufacture the paper microzone plates.

\section{Instrumentation}

An infrared camera (7.5-13.0 $\mu$ m, model FLIR E60, FLIR, USA) was used for temperature monitoring in TIE analysis. It provided images with $320 \times 240$ pixels at the frame rate of $30 \mathrm{~Hz}$, which were recorded and processed using ResearchIR software (version 3.5, FLIR). A wax printer (ColorQube, 8870, Xerox, USA) and an oven (MA 033/100, Marconi, Brazil) were used to prepare the paper microzone plates, while an electronic multichannel pipette (eight channels, 15 to $300 \mu \mathrm{L}$, Xplorer, Eppendorf, Germany) was used for reagent addition and a vortex mixer (MS-X, Scilogex, USA) for homogenization. An ultrasound bath was used to remove carbon dioxide from sparkling wine (TI-H-5, $25 \mathrm{kHz}$, Elma, Germany). Total acidity determination by the reference AOAC method was performed by potentiometric titration using a potentiometer and combined glass electrode (DM- 
22, Digimed, Brazil). For fixed acidity determination via the reference AOAC method, a water bath (SL 152/10, Solab, Brazil) was used for heating. The paper surface micrograph was obtained using a scanning electron microscope (SEM) (model Sigma 300 VP, Carl Zeiss, England) using a field emission gun Schotky type (tungsten filament coated with zirconium oxide) equipped with Gemini column. A power meter (43B model, Fluke Corporation, USA) was used for measuring the energy consumption. The statistical analyses were performed using Statistica 7.0 software. ${ }^{23}$

Acidities determination by reference method (AOAC International, Method 962.12)

Sparkling wine samples were previously sonicated for 10 min to eliminate carbon dioxide. Red and white wines were analyzed directly without any pretreatment, and total acidity determination was performed by potentiometric titration using a $0.1 \mathrm{~mol} \mathrm{~L}^{-1}$ sodium hydroxide solution as titrant and $10 \mathrm{~mL}$ of sample plus $50 \mathrm{~mL}$ of water. ${ }^{12}$ For fixed acidity determination, $10 \mathrm{~mL}$ of each sample were inserted into porcelain capsules and heated in a boiling water bath for evaporation. This procedure was repeated three times, reaching a total volume of $30 \mathrm{~mL}$ of sample for each determination. Afterwards, the residue was diluted in $50 \mathrm{~mL}$ of water and the same procedure described above for total acidity determination was followed. Volatile acidity was obtained by difference between total and fixed acidity. All analyses were performed in triplicate, and the results were presented in tartaric acid concentration $\left(\mathrm{mEq} \mathrm{L}^{-1}\right)$.

Acidities determination by the proposed TIE method

The paper microzone plates were designed under the same dimensions of polystyrene microplate used in TIE with
24 zones (plate dimension $123 \times 81 \mathrm{~mm}$, length $\times$ width; zone diameter $16 \mathrm{~mm}$; zone-to-zone distance $2 \mathrm{~mm}$ ). ${ }^{16}$ The layout was printed on paper using a wax printer. Afterwards, the paper was heated at $105^{\circ} \mathrm{C}$ in an oven for 5 min in order to melt the wax so that a hydrophobic barrier could be formed. ${ }^{10,24}$ These paper plates were adapted into a plastic support to avoid the analytical zones coming into direct contact with the workbench. Figure 1 represents the approach used in the proposed method.

An initial study on the influence of mixing solutions was performed, focusing on the volume and the dispensing rate of reagent solution. In this way, volumes between 30 to $70 \mu \mathrm{L}$ of solutions and dispensing rates between 40 to $400 \mu \mathrm{L} \mathrm{s}^{-1}$ were evaluated, using $0.05 \mathrm{~mol} \mathrm{~L}^{-1}$ tartaric acid and $2 \mathrm{~mol} \mathrm{~L}^{-1}$ sodium hydroxide solution as a reagent in stoichiometric excess. A ratio of 1:1 was chosen for all experiments based on the previous results for TIE. ${ }^{19}$ Results were evaluated considering the relative standard deviation (RSD, in percentage) of measurements.

The infrared camera was positioned on a tripod $40 \mathrm{~cm}$ above the plate to perform the measurements. Room temperature, relative humidity, and emissivity were adjusted as recommended by the camera manufacturer. Using the software, the temperature of each microzone was obtained with a circle tool for measuring different ROI (from 61 to 349 pixels). The average temperature was plotted over time, forming an enthalpogram. The temperatures $2 \mathrm{~s}$ before the addition of the reagent and $2 \mathrm{~s}$ after were considered to obtain the difference of temperature $(\Delta \mathrm{T})$, obtained from equation $\Delta T=T_{f}-T_{i}$, where $T_{f}$ is final temperature and $T_{i}$ initial temperature. The acidities values were obtained using the equation from the calibration curve. In all experiments, the 24 zones of plate $(n=24)$ were considered.

Calibration curves were constructed using reference solutions with concentration ranging from 0.025 to

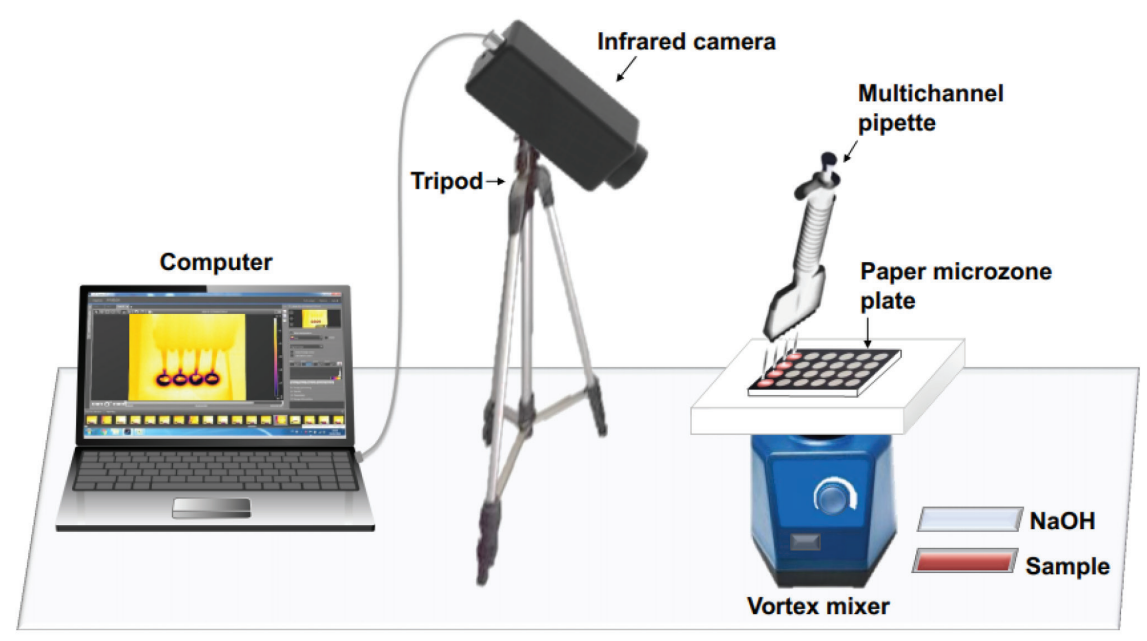

Figure 1. Apparatus setup used for the determination of wine acidities in paper microzone plates. 
$0.175 \mathrm{~mol} \mathrm{~L}^{-1}$ of tartaric acid $(\mathrm{n}=24)$. The experimental procedure used was the addition of tartaric acid (for curve construction) or sample solutions into the microzones, followed by the addition of $2 \mathrm{~mol} \mathrm{~L}^{-1}$ sodium hydroxide solution. Afterwards, the paper plate was immediately shaken for $2 \mathrm{~s}$ using a vortex mixer set in the minimum speed. All the blanks were performed using water instead of sample or reference solutions.

For the fixed acidity determination, the temperature of evaporation in the oven was evaluated from 60 to $100{ }^{\circ} \mathrm{C}$. After determining the most suitable temperature $\left(80^{\circ} \mathrm{C}\right)$, $50 \mu \mathrm{L}$ from each sample were added into the plate and evaporated in the oven for $10 \mathrm{~min}$. This procedure was repeated three times, reaching a total of $150 \mu \mathrm{L}$ of sample concentrated in the microzones. The plates were placed in the desiccator until they reached room temperature (around $25^{\circ} \mathrm{C}$ ). After, a reconstitution with $50 \mu \mathrm{L}$ of water was performed and the same procedure used to analyze total acidity was followed.

\section{Scanning electron microscopy (SEM)}

Paper microzone plates were evaluated using SEM to observe any difference in wax and the surface of the paper. Additionally, the latter was evaluated after 1,3, and 7 cycles of concentration to demonstrate the behavior of the sample within the paper structure. The microscopic evaluations were performed in high resolution (up to $1 \mathrm{~nm}$ ) with a high vacuum mode $\left(1 \times 10^{-9}\right.$ bar $)$ according to the equipment's manufacturer. The images were obtained using a secondary electron detector in high vacuum mode.

\section{Results and Discussion}

The enthalpogram obtained from analytical signal is shown Figure 2. Initially, the sample was added on the microzone and $2 \mathrm{~s}$ was selected for $\mathrm{T}_{\mathrm{i}}$ determination. Afterwards, the addition of sodium hydroxide caused a temperature rise resulted from the chemical reaction. A stirring for $2 \mathrm{~s}$ was applied for improving the homogenization of solution and for obtaining a suitable measurement of temperature. After the homogenization step, the $\mathrm{T}_{\mathrm{f}}$ was selected in the region of stabilization of analytical signal. Thus, it can be seen that the complete reaction on paper surface took only $10 \mathrm{~s}$.

\section{Evaluation of experimental parameters}

The pipetting and temperature measurement conditions were evaluated in order to reduce the deviations among measurements before quantitative analyses. Pipetting was

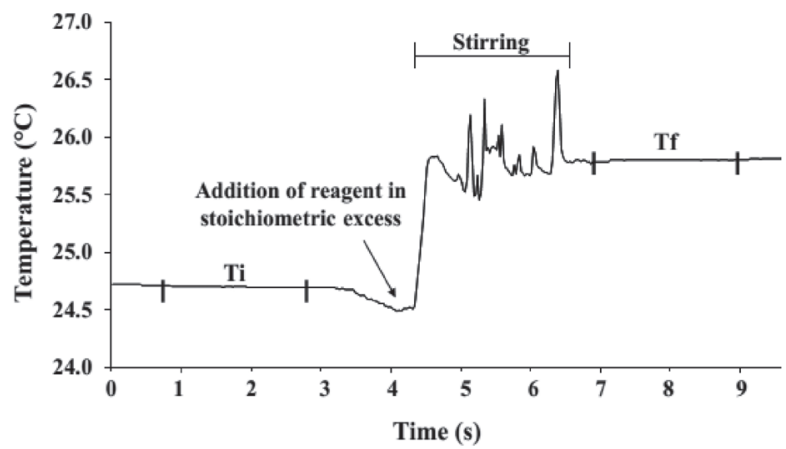

Figure 2. Analytical signal obtained by neutralization reaction, where $T_{i}$ is the initial temperature and $T_{f}$ is the final temperature. The reaction was performed with reference solutions of $0.5 \mathrm{~mol} \mathrm{~L}^{-1}$ tartaric acid and 2 mol L-1 $\mathrm{NaOH}$.

evaluated in different volumes ( 30 to $70 \mu \mathrm{L}$ ) and dispensing rates (40 to $400 \mu \mathrm{L} \mathrm{s}^{-1}$ ). As shown in Figure 3, the most suitable conditions were $50 \mu \mathrm{L}$ of volume of each solution and $200 \mu \mathrm{L} \mathrm{s}^{-1}$ of dispensing rate, because they allowed uniform mixing and provided lower RSD (around 0.32\%) among measurements. The optimization showed that lower volumes (e.g., 30 and $40 \mu \mathrm{L}$ ) do not completely fill the detection zones, resulting in high deviations. On the other hand, the use of higher volumes led to the loss of solutions from the microzones.

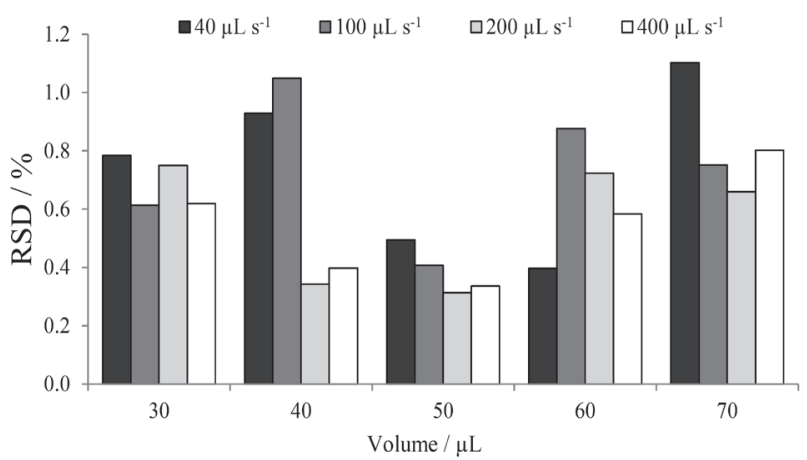

Figure 3. Results obtained from optimization of the volume and dispensation rates of reagent solution in stoichiometric excess $\left(\mu \mathrm{L} \mathrm{s}^{-1}\right)$ for wine acidity determination by TIE $(n=24)$. Reactions were performed with reference solutions of $0.5 \mathrm{~mol} \mathrm{~L}^{-1}$ tartaric acid and $2 \mathrm{~mol} \mathrm{~L}^{-1} \mathrm{NaOH}$.

In relation to the dispensing rate, a lower variation of results using $200 \mu \mathrm{L} \mathrm{s}^{-1}$ was observed with suitable distribution of the solution over the analytical zone. Considering that the paper microzone plates are exposed to the environment and the reaction occurs in an instantaneous way after addition of the excess reagent, a lower rate of dispensing could result in heat loss or unsuitable homogenization. However, higher dispensing rates (e.g., $400 \mu \mathrm{L} \mathrm{s}^{-1}$ ) resulted in liquid projection out of the analytical zone, providing higher variation among measurements.

The image processing was also evaluated for the determination of temperature considering the ROI 
(represented by different number of pixels). Considering that each analytical zone has $16 \mathrm{~mm}$ of diameter, a circle with 349 pixels was enough to cover the whole reaction area. As can be seen in Figure 4, lower RSD values were obtained until 129 pixels, but for higher ROI the RSD increased. This probably occurred because when the solution is very close to the borders of analytical zones, where heat dissipation is higher, this causes temperature variations due to contact with the wax. As such, we did not observe a significant difference between 61 and 97 pixels with RSD of 0.63 and $0.58 \%$, respectively. These number of pixels were equivalent to a circle with diameter of 5 and $6 \mathrm{~mm}$ in the center of analytical zone. However, the area correspondent to 97 pixels was considered the most suitable, once it is more representative, and for this reason it was selected for subsequent experiments.

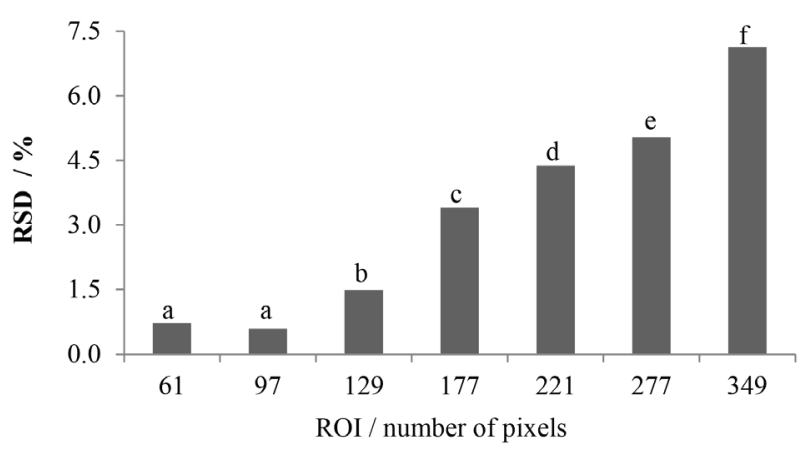

Figure 4. Evaluation of the ROI (reported in number of pixels) used for temperature measurement. Reactions were performed with reference solutions of $0.5 \mathrm{~mol} \mathrm{~L}^{-1}$ tartaric acid and $2 \mathrm{~mol} \mathrm{~L}^{-1} \mathrm{NaOH}(\mathrm{n}=24)$. Means followed by the same letters did not differ $(p>0.05)$ by Tukey's test.

\section{Determination of wine acidities}

A calibration curve was constructed for acidities determination using TIE with solutions of tartaric acid in concentrations varying from 0.025 to $0.175 \mathrm{~mol} \mathrm{~L}^{-1}$, as shown in the Supplementary Information section (Figure S1).

In the determination of fixed acidity, samples were firstly evaporated under different temperatures (60, 70, 80,90 , and $100{ }^{\circ} \mathrm{C}$ ) in order to reach shorter time. As expected, we observed a reduction of drying time with the temperature increase, and the total times (three cycles) required to concentrate samples were 50, 42, 30, 26, and $20 \mathrm{~min}$ for $60,70,80,90$, and $100{ }^{\circ} \mathrm{C}$, respectively. However, the application of higher temperatures (90 and $100{ }^{\circ} \mathrm{C}$ ) led to changes in paper appearance, and so the chosen temperature for sample evaporation was $80{ }^{\circ} \mathrm{C}$. It is important to highlight that in this temperature, the evaporation step required for fixed acidity determination was performed for only $30 \mathrm{~min}$ for TIE, while for the reference AOAC method 150 min were required. Drying in an oven facilitates the simultaneous drying of a larger number of samples (hundreds) using paper microzone plates and thus enables an increase in sample throughput, unlike the reference AOAC method.

Figure 5 shows the microcopies for the paper surface, which consists in irregular cellulose fibers with rough and porous structures (Figure 5A). Before heating (Figure 5B), the wax completely covers the paper surface, but after heating it diffuses throughout the paper to form the hydrophobic barrier (Figure 5C). The compounds presented in the sample added on the paper are retained, and after each cycle of evaporation, the sample accumulated in the paper with an increase of material after successive cycles of concentration (Figures $5 \mathrm{D}, 5 \mathrm{E}$, and $5 \mathrm{~F})$. Thus, it was possible to concentrate the sample directly on the paper plate. In spite of the possibility of concentration showed by microscopies, the influence of such cycles in the analytical response was also performed. As shown in Figure 6, the number of sample concentration on the paper presented proportional results to the increase of temperature. Thus, a fast sample evaporation could be used for concentration of analytes and allow the determination of acidities for a wide range of wines. However, the results indicate that it can be applied for analyzing sample with low acidity, once up to 7 concentration cycle there was no loss of analyte on the paper. For analysis of fixed acidity, the sample was concentrated in three cycles in order to achieve a suitable analytical signal.

Afterwards, the determination of total and fixed acidities of wine samples were carried out, and the results are presented in Table 1. For the three different wines evaluated in this work, good agreements of the results of TIE with the reference AOAC method (from 97 to $101 \%$ for total acidity, 100 to $104 \%$ for fixed acidity, and 96 to $103 \%$ for volatile acidity) were observed. Although the evaluated wines present different chemical compositions, no influence on the performance of analyses was found, which can be considered an indication of the robustness of the proposed method even for dark colored samples as red wines, because TIE is based on infrared rather than visible detection. According to recommendations by the Brazilian authorities, ${ }^{25}$ the wines should have a total acidity of between 55 to $130 \mathrm{mEq} \mathrm{L}^{-1}$ and a maximum volatile acidity of $20 \mathrm{mEq} \mathrm{L}{ }^{-1}$. Therefore, all samples evaluated could be considered to be in agreement with official regulations.

For all analyzed samples, the results obtained with the TIE method presented a reduction in the deviation among measurements, probably due to the reduction of analytical operations and the possibility to use large number of replicates in a simple manner $(n=24)$. The TIE method 


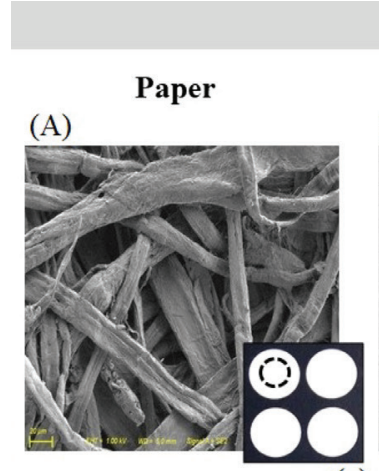

(a)

\section{WITHOUT SAMPLE}

(B)

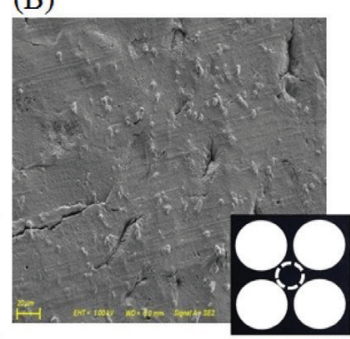

Paper + melted wax

(C)

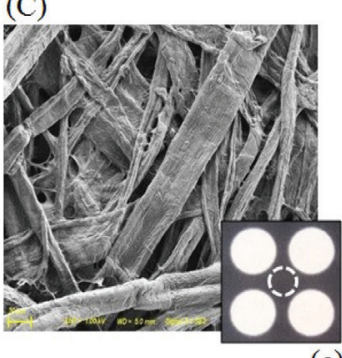

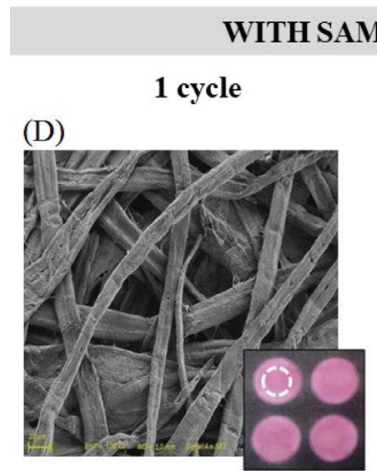

(d) (b) (c)

\begin{abstract}
PLE (number of concentration cycles)
\end{abstract}
3 cycles

(E)

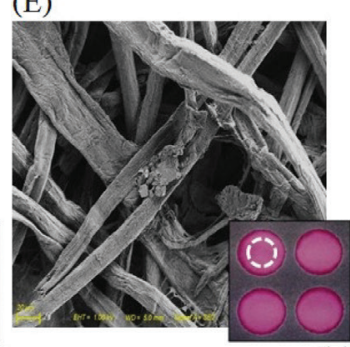

7 cycles

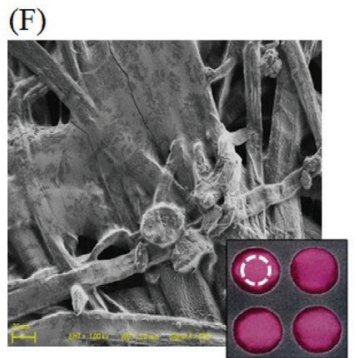

(e)

(f)

Figure 5. Scanning electron micrographs and visible images of paper microzone without and with the sample (representing the number of wine concentration cycles on the paper). Figures from (A) to (F) and (a) to (f) are related to microscopy (500x) and visible images of paper microzone plates, respectively. Dashed lines in the images from (a) to (f) represent the area of the paper microzone plates evaluated in microscopy.

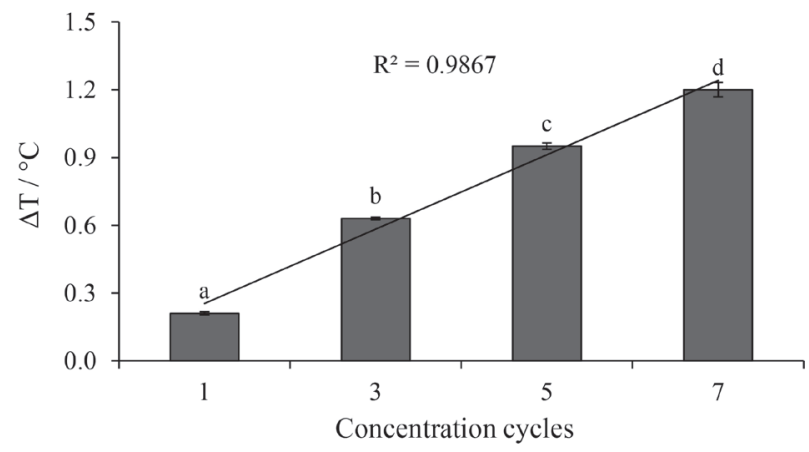

Figure 6. Influence of the number of concentration cycles in the temperature obtained for the determination of fixed acidity in the red wine sample (mean \pm standard deviation, $\mathrm{n}=24$ ).

allows the possibility to perform the evaporation and determination steps in the same analytical zone, avoiding excessive handling and analyte losses, which lead to lower deviations.

\section{Parameters of merit of the proposed method}

In Table 2, a comparison of the parameters of merit was performed for three different methods to determine acidity: conventional TIE (performed in polystyrene plate), TIE in paper microzone plates, and reference AOAC titration. The results obtained for conventional TIE were not performed by this work, as they were completely based on data from the literature. ${ }^{17}$

In general, TIE performed in paper microzone plates presented very satisfactory results, with important advantages for all evaluated parameters, mainly in relation to the titration method. By using TIE in paper microzone plates, it was possible to obtain a reduction in reagent and sample consumption, reaching sample consumption 200 times lower than reference AOAC titration method. In addition, the consumption of sodium hydroxide was also significant from about one order of magnitude, showing that TIE based on paper allowed the reduction of the amount of reagent used and waste generated. It is important to mention that the wax printing is considered an eco-friendly method for preparation of paper microzone plates because the solid ink deposited on the paper is formulated from a polymer based on nontoxic resin. ${ }^{5}$ Therefore, taking into account that paper devices are biodegradable and obtained from renewable resources, they make the proposed method even more suitable when attempting to meet the principles of green analytical chemistry.

For determination of fixed acidity, the sample evaporation on paper showed to be faster, decreasing from 120 to $30 \mathrm{~min}$, mainly due to a smaller sample volume and 
Table 1. Results (mean \pm standard deviation) for total, fixed, and volatile acidities for different wines using TIE in paper microzone plates $(\mathrm{n}=24)$ and reference titration $(\mathrm{n}=3$, AOAC) methods

\begin{tabular}{|c|c|c|c|c|c|c|c|}
\hline \multirow{2}{*}{ Sample } & \multirow{2}{*}{ Manufacturer } & \multicolumn{2}{|c|}{ Total acidity / $\left(\mathrm{mEq} \mathrm{L}{ }^{-1}\right)$} & \multicolumn{2}{|c|}{ Fixed acidity / $\left(\mathrm{mEq} \mathrm{L}^{-1}\right)$} & \multicolumn{2}{|c|}{ Volatile acidity / $\left(\mathrm{mEq} \mathrm{L}^{-1}\right)$} \\
\hline & & AOAC & TIE, proposed & AOAC & TIE, proposed & AOAC & TIE, proposed \\
\hline & 1 & $86.0 \pm 1.6$ & $85.3 \pm 0.8$ & $74.0 \pm 1.5$ & $73.4 \pm 0.4$ & $11.0 \pm 2.2$ & $12.0 \pm 0.9$ \\
\hline \multirow[t]{3}{*}{ Red wine } & 2 & $94.1 \pm 1.9$ & $93.7 \pm 0.8$ & $79.0 \pm 1.3$ & $78.7 \pm 0.7$ & $15.1 \pm 2.3$ & $15.1 \pm 1.0$ \\
\hline & 3 & $98.9 \pm 1.9$ & $99.0 \pm 0.9$ & $80.6 \pm 2.8$ & $80.0 \pm 0.5$ & $18.3 \pm 3.4$ & $19.0 \pm 1.1$ \\
\hline & 1 & $85.0 \pm 1.6$ & $85.2 \pm 0.7$ & $72.0 \pm 1.9$ & $72.7 \pm 0.7$ & $13.0 \pm 2.5$ & $12.5 \pm 1.0$ \\
\hline \multirow[t]{3}{*}{ Sparkling wine } & 2 & $68.7 \pm 1.6$ & $68.3 \pm 0.6$ & $54.7 \pm 1.8$ & $54.7 \pm 0.7$ & $14.0 \pm 2.4$ & $13.7 \pm 0.9$ \\
\hline & 3 & $80.9 \pm 1.4$ & $80.4 \pm 0.7$ & $68.0 \pm 2.1$ & $67.3 \pm 0.6$ & $12.9 \pm 2.5$ & $13.1 \pm 1.0$ \\
\hline & 1 & $70.3 \pm 1.7$ & $70.8 \pm 0.8$ & $54.0 \pm 1.5$ & $54.0 \pm 0.7$ & $16.3 \pm 2.3$ & $16.8 \pm 1.1$ \\
\hline \multirow[t]{2}{*}{ White wine } & 2 & $90.0 \pm 1.5$ & $89.3 \pm 0.8$ & $70.7 \pm 2.5$ & $70.7 \pm 0.7$ & $19.4 \pm 2.9$ & $18.7 \pm 1.1$ \\
\hline & 3 & $86.9 \pm 1.6$ & $86.5 \pm 0.9$ & $77.5 \pm 1.5$ & $77.0 \pm 0.6$ & $9.4 \pm 2.1$ & $9.6 \pm 1.1$ \\
\hline
\end{tabular}

AOAC: AOAC International; TIE: thermal infrared enthalpimetry.

Table 2. Comparison of the parameters of merit in the determination of total and fixed acidity by TIE in paper microzone plates, TIE in microplates, ${ }^{17}$ and reference titration (AOAC) methods

\begin{tabular}{|c|c|c|c|c|c|c|}
\hline \multirow[b]{2}{*}{ Parameter } & \multicolumn{3}{|c|}{ Total acidity } & \multicolumn{3}{|c|}{ Fixed acidity } \\
\hline & TIE, proposed & $\begin{array}{l}\text { TIE, polymeric } \\
\text { microplate }^{17}\end{array}$ & $\mathrm{AOAC}$ & TIE, proposed & $\begin{array}{l}\text { TIE, polymeric } \\
\text { microplate }^{17}\end{array}$ & $\mathrm{AOAC}$ \\
\hline Volume of sample $/ \mathrm{mL}$ & 0.05 & 1.2 & 10 & 0.15 & 7 & 30 \\
\hline Reagent consumption ${ }^{\mathrm{a}}(\mathrm{NaOH}) /(\mathrm{mg}$ per sample) & 4 & 96 & $30-90$ & 4 & 96 & $15-23$ \\
\hline time / (min per sample) & 3 & 3 & 10 & 33 & 120 & 120 \\
\hline Sample throughput / (sample $\left.\mathrm{h}^{-1}\right)$ & 480 & 480 & 18 & $120^{\mathrm{b}}$ & $36^{\mathrm{b}}$ & $1.5^{\mathrm{b}}$ \\
\hline Energy consumption / (kW h per sample) & $0.001^{\mathrm{c}}$ & $0.001^{\mathrm{d}}$ & $0.037^{\mathrm{e}}$ & $0.001^{\mathrm{cf}}$ & $1.49^{\mathrm{dg}}$ & $15.90^{\mathrm{ef}}$ \\
\hline
\end{tabular}

${ }^{a}$ Calculation was performed considering only one measurement; ${ }^{b}$ considering ten microplates for proposed TIE, three microplates in one water bath in each run for both TIE in polymeric microplate ${ }^{17}$ and for AOAC; ${ }^{c}$ considering the multichannel pipette, infrared (IR) camera, and vortex; ${ }^{\mathrm{d}}$ considering the multichannel pipette, IR camera, and magnetic stirrer; ${ }^{\mathrm{e}}$ considering the potentiometer; ${ }^{\mathrm{f}}$ considering the heating oven; ${ }^{\mathrm{g}} \mathrm{considering}$ the thermostatic bath. TIE: thermal infrared enthalpimetry; AOAC: AOAC International.

high surface to volume ratio of the paper. Additionally, as the sample evaporation in paper is performed in an oven, it can be an appropriate procedure for routine activities, allowing simultaneous concentration of at least 50 plates. This is equivalent to 1200 samples processed per cycle of heating. In the reference AOAC method carried out in a water bath, it was possible to concentrate only three samples simultaneously. Another feature of the proposed TIE method is the possibility to perform the determination step in only $3 \mathrm{~min}$ for 24 samples. Due to this shorter time, TIE presented high throughput, reaching 120 samples per $\mathrm{h}$ for fixed acidity and 480 for total acidity.

Despite the advantages cited above, the energy consumption determined for the three methods again indicated more favorable results for TIE performed in paper, with lower energy consumption for both acidity determinations. TIE performed in paper microzone plates spent $0.001 \mathrm{~kW} \mathrm{~h}$ for fixed acidity and $0.0006 \mathrm{~kW}$ h for total acidity, while for conventional TIE (in polystyrene microplates) $0.66 \mathrm{~kW} \mathrm{~h}$ were consumed for fixed acidity and $0.001 \mathrm{~kW}$ h for total acidity. For reference AOAC titration method, the energy consumption was $15.87 \mathrm{~kW} \mathrm{~h}$ for fixed acidity, showing a clear positive impact of the proposed method.

\section{Conclusions}

The combination of TIE with paper microzone plates may be considered a viable and fast alternative for acidities evaluation of wine, showing accurate and precise results. It is important to mention what differences, either in chemical composition or in the intense color (red wine), do not influence the method performance. A reduction in the use of sample and reagent amount, lower energy consumption, and higher sample throughput could be observed in relation to the reference AOAC titration method. 


\section{Supplementary Information}

Supplementary data (e.g., typical calibration curve) are available free of charge at http://jbcs.sbq.org.br as PDF file.

\section{Acknowledgments}

Authors thank Prof Edson I. Muller and Michele S. P. Enders for microscopies. This study was financed in part by the Coordenação de Aperfeiçoamento de Pessoal de Nível Superior (CAPES, Brazil, Finance Code 001). The authors also thank the Conselho Nacional de Desenvolvimento Científico e Tecnológico (CNPq, Brazil) and the Fundação de Amparo à Pesquisa do Estado do Rio Grande do Sul (FAPERGS) for supporting this study.

\section{References}

1. Tobiszewski, M.; Namieśnik, J.; Ecotoxicol. Environ. Saf. 2015, 120, 173.

2. Clark, J. H.; Green Chem. 1999, 1,1 .

3. Gałuszka, A.; Migaszewski, Z.; Namieśnik, J.; TrAC, Trends Anal. Chem. 2013, 50, 78.

4. Songjaroen, T.; Dungchai, W.; Chailapakul, O.; Laiwattanapaisal, W.; Talanta 2011, 85, 2587.

5. Costa, M. N.; Veigas, B.; Jacob, J. M.; Santos, D. S.; Gomes, J.; Baptista, P. V.; Martins, R.; Inácio, J.; Fortunato, E.; Nanotechnology 2014, 25, 094006.

6. Morbioli, G. G.; Mazzu-Nascimento, T.; Stockton, A. M.; Carrilho, E.; Anal. Chim. Acta 2017, 970, 1.

7. Martinez, A. W.; Phillips, S. T.; Whitesides, G. M.; Carrilho, E.; Anal. Chem. 2010, 82, 3.

8. Vaher, M.; Kaljurand, M.; Anal. Bioanal. Chem. 2012, 404, 627.

9. Mazzu-Nascimento, T.; Morbioli, G. G.; Milan, L. A.; Silva, D. F.; Donofrio, F. C.; Mestriner, C. A.; Carrilho, E.; Anal. Methods 2017, 9, 2644.

10. Carrilho, E.; Martinez, A. W.; Whitesides, G. M.; Anal. Chem. 2009, 81, 7091 .
11. Carrilho, E.; Phillips, S. T.; Vella, S. J.; Martinez, A. W.; Whitesides, G. M.; Anal. Chem. 2009, 81, 5990.

12. Horwitz, W.; Latimer, G. W.; Official Methods of Analysis of AOAC International, $8^{\text {th }}$ ed.; AOAC International, Gaithersburg, USA, 2005.

13. Karita, S.; Kaneta, T.; Anal. Chem. 2014, 86, 12108.

14. Taghizadeh-Behbahani, M.; Hemmateenejad, B.; Shamsipur, M.; Chem. Pap. 2018, 72, 1239.

15. Nogueira, S.; Sousa, L.; Silva, N.; Rodrigues, P.; Coltro, W.; Micromachines 2017, 8, 139.

16. Barin, J. S.; Tischer, B.; Oliveira, A. S.; Wagner, R.; Costa, A. B.; Flores, E. M. M.; Anal. Chem. 2015, 87, 12065.

17. Tischer, B.; Oliveira, A. S.; Ferreira, D. F.; Menezes, C. R.; Duarte, F. A.; Wagner, R.; Barin, J. S.; Food Chem. 2017, 215, 17.

18. Tischer, B.; Oliveira, A. S.; Costa, A. B.; Cichoski, A. J.; Barcia, M. T.; Wagner, R.; Barin, J. S.; J. Food Compos. Anal. 2017, 63,34 .

19. Oliveira, A. S.; Dalla Nora, F. M.; Mello, R. O.; Mello, P. A.; Tischer, B.; Costa, A. B.; Barin, J. S.; Talanta 2017, 171, 335.

20. Oliveira, A. S.; Ballus, C. A.; Menezes, C. R.; Wagner, R.; Paniz, J. N. G.; Tischer, B.; Costa, A. B.; Barin, J. B.; Food Chem. 2018, 258, 59.

21. Dalla Nora, F. M.; Oliveira, A. S.; Lucas, B. N.; Ferreira, D. F.; Duarte, F. A.; Mello, R. O.; Cichoski, A. J.; Barin, J. S.; Anal. Methods 2018, 10, 3770.

22. Alessio, K. O.; Voss, M.; Flores, E. M. M.; Costa, A. B.; Duarte, F. A.; Barin, J. S.; Talanta 2019, 204, 266.

23. Statistica 7.0; StatSoft Inc., Tulsa, USA, 2009.

24. Lo, S.-J.; Chen, K.-H.; Yao, D.-J.; Talanta 2015, 145, 29.

25. Ministério da Agricultura, Pecuária e Abastecimento (MAPA); Portaria No. 229, 25 de Outubro de 1988; Complementação de Padrões de Identidade e Qualidade de Vinho; Diário Oficial da União, Brasília, 31 de Outubro de 1988. Available at http://www. agricultura.gov.br/assuntos/vigilancia-agropecuaria/ivegetal/ bebidas-arquivos/portaria-no-229-de-25-de-outubro-de-1988. pdf/view, accessed in August 2019.

Submitted: April 19, 2019

Published online: August 20, 2019 